\section{(C) OPEN ACCESS}

\title{
Occupational biomechanical risk factors for radial nerve entrapment in a 13-year prospective study among male construction workers
}

\author{
Jennie A Jackson, ${ }^{\oplus}$ David Olsson, ${ }^{1}$ Alex Burdorf, ${ }^{2}$ Laura Punnett, ${ }^{3}$ Bengt Järvholm, ${ }^{1}$ \\ Jens Wahlström ${ }^{1}$
}

- Additional material is published online only. To view please visit the journal online (http://dx.doi.org/10.1136/ oemed-2018-105311).

${ }^{1}$ Department of Public Health and Clinical Medicine, Umeå University, Umeå, Sweden ${ }^{2}$ Department of Public Health, Erasmus MC, Rotterdam, The Netherlands

${ }^{3}$ Department of Biomedical Engineering, University of Massachusetts Lowell, Lowell, Massachusetts, USA

\section{Correspondence to} Dr Jennie A Jackson Occupational and Public Health Sciences, Hogskolan i Gavle, Gavle 801 76, Sweden; jennie.jackson@hig.se

Received 6 November 2018 Revised 14 February 2019 Accepted 18 February 2019 Published Online First 8 March 2019

\begin{abstract}
Objectives The aim was to assess the association between occupational biomechanical exposure and the occurrence of radial nerve entrapment (RNE) in construction workers over a 13-year follow-up period. Methods A cohort of 229707 male construction workers who participated in a national occupational health surveillance programme (1971-1993) was examined prospectively (2001-2013) for RNE. Height, weight, age, smoking status and job title (construction trade) were obtained on health examination. RNE case status was defined by surgical release of RNE, with data from the Swedish national registry for out-patient surgery records. A job exposure matrix was developed, and biomechanical exposure estimates were assigned according to job title. Highly correlated exposures were summed into biomechanical exposure scores. Negative binomial models were used to estimate the relative risks (RR) (incidence rate ratios) of RNE surgical release for the biomechanical factors and exposure sum scores. Predicted incidence was assessed for each exposure score modelled as a continuous variable to assess exposure-response relationships.
\end{abstract}

Results The total incidence rate of surgically treated RNE over the 13-year observation period was 3.53 cases per 100000 person-years. There were 92 cases with occupational information. Increased risk for RNE was seen in workers with elevated hand-grip forces ( $R R=1.79,95 \% \mathrm{Cl} 0.97$ to 3.28$)$ and exposure to handarm vibration ( $R R=1.47,95 \% \mathrm{Cl} 1.08$ to 2.00$)$. Conclusions Occupational exposure to forceful handgrip work and vibration increased the risk for surgical treatment of RNE.

\section{INTRODUCTION}

All three major peripheral nerves to the forearm and hand-the median, ulnar and radial nervestraverse the elbow joint, then weave between and around muscles, blood vessels and other soft tissue structures in the forearm, making them vulnerable to compression. The most common nerve entrapment syndromes in the forearm are carpal tunnel syndrome (CTS), cubital tunnel syndrome and radial tunnel syndrome (RTS), resulting from compression in the forearm of the median, ulnar and radial nerves, respectively. Of these, radial nerve entrapment (RNE) is by far the least studied and most controversial in terms of diagnosis and surgical treatment. RNE in the forearm can result

\section{Key messages}

What is already known about this subject?

- Occupational biomechanical exposures such as repetitive movements, vibration and elevated grip force may increase the risk for radial nerve entrapment (RNE).

\section{What are the new findings?}

- Workers with elevated hand-grip forces and with exposure to hand-arm vibration were at higher risk of surgery for RNE. Workers exposed to repetitive elbow and wrist flexion and extension showed a modest association with risk of surgery for RNE.

\section{How might this impact on policy or clinical practice in the foreseeable future? \\ - Decreased exposure to occupational biomechanical risk factors may be important for primary prevention as well as secondary and tertiary prevention for persons with RNE.}

not only in RTS but also posterior interosseous nerve syndrome (PINS). RTS is typically used to describe the physiological symptoms resulting from RNE when no motor function is lost and is thought to result from compression of the radial nerve in the radial tunnel of the elbow by one or more of the following structures: arcade of Frohse; tendinous margins of extensor carpi radialis brevis; leash of Henry (comprised vessels from the radial recurrent artery); or fibrous bands distal to the radial head. ${ }^{1}$ While RTS is relatively rare, ${ }^{2}$ it can lead to functional limitations that impact both quality of life and work ability. The radial nerve in the forearm innervates aspects of the thumb, index, middle and ring fingers, giving it a key role in gripping and dexterity. Primary symptoms include aching muscle pain in the dorsal forearm, ${ }^{34}$ tiredness, ${ }^{3}$ tenderness or pain over the radial tunnel,,$^{5}$ and discomfort during resisted forearm supination and/or middlefinger extension. ${ }^{6}$ PINS differs from RTS in that patients also experience loss of motor function and pain is not the primary symptom. ${ }^{3}$ PINS is thought to result from compression of the posterior interosseous nerve distal to the bifurcation of the radial nerve. $^{7}$ 
RTS has been described in different occupational groups including industrial textile workers, ${ }^{4}$ television, shoe and automobile manufacturing workers, ${ }^{5}$ brick layers, fitters, machine operators and telephonists. ${ }^{8}$ One case-control study examined biomechanical exposures and identified repetitive grip force work (OR 9.1, 95\% CI 1.4 to 56.9), prolonged static hand loading (OR 5.9, 95\% CI 1.2 to 29.9) and working with fully extended elbows (OR 4.9, 95\% CI 1.0 to 25.0 ) as occupational risk factors for RTS. ${ }^{5}$ The study was based on 21 cases and 21 controls ( 17 women and 4 men) and thus had low power leading to low precision in risk estimate. Occupational exposures were assigned based on expert opinions on the job held 6 months prior to diagnosis but did not consider the influence of a longer exposure history.

Diagnosis of RNE can be difficult and is often controversial. The use of strict inclusion criteria can limit the risk of misclassified cases, including cases of epicondylitis. ${ }^{5}$ Patients with RNE who do not respond to rest, anti-inflammatory medication or physiotherapy exercises are candidates for surgical decompression of the radial nerve, distal to the elbow ${ }^{3}$ and thus the use of a surgical case definition for RNE could decrease misclassification.

The aim of the current study was therefore to assess the association between historical information on biomechanical exposure and the occurrence of decompression surgery for RNE in a large construction worker cohort over a 13-year follow-up period.

\section{METHODS}

A large sample of male construction workers was followed prospectively for 13 years to identify the surgical treatment of RNE and its association with prior occupational, physical exposures.

\section{Data}

The study cohort was selected from a total of 389132 Swedish construction workers who participated in health examinations as part of a national health surveillance programme ('Bygghälsan') conducted from the late 1960s until 1993. While participation was voluntary, at least $80 \%$ of eligible workers completed at least one health examination. ${ }^{9}$ Worker height, weight, age, smoking status and specific trade ('job title') data were recorded on examination.

The Swedish national registry of outpatient surgical records was searched to determine cases, defined by surgical release of RNE (Swedish code ACC52). For this study, computerised data were available from the start of the register in 2001 until the end of 2013. Register linkage was achieved using the unique personal number assigned to each Swedish resident.

Worker job titles were classified into 21 occupational groups defined by occupational health service experts at the time of the surveillance programmes. Occupational groups comprised jobs with similar tasks and workers with similar background training. A group was also made for unclassifiable jobs. Full details of the job-to-group mappings were previously presented. ${ }^{10}$

Biomechanical exposure levels were assigned to occupational groups using a job exposure matrix (JEM) developed for the study. The JEM contained 12 exposure factors that were deemed a priori to be aetiologically relevant based on the available literature on upper extremity disorders (table 1 ). Two experts reviewed ergonomic assessments conducted in the 1970s for each job title (described in detail in Jackson $e a^{10}{ }^{10}$ ) and independently rated the average exposure intensity or frequency over a working day across all job titles comprising each occupational group and for each JEM factor. All ratings were done blinded to the number of
Table 1 Biomechanical risk factors included in the JEM

\begin{tabular}{ll}
\hline Exposure & Rating \\
\hline Intensity of handgrip force & $1-3^{*}$ \\
\hline Intensity of upper extremity load & $1-3^{*}$ \\
\hline Frequency of repetitive elbow flexion and extension work & $1-3^{*}$ \\
\hline Frequency of repetitive wrist flexion and extension work & $1-3^{*}$ \\
Frequency of hand-held tool use & $1-3^{*}$ \\
\hline Frequency of upper extremity static work & $1-3^{*}$ \\
Frequency of full wrist extension & $1-3^{*}$ \\
\hline Frequency of full elbow extension & $1-3^{*}$ \\
Frequency of using a hand-held tool in a fixed position & $1-3^{*}$ \\
\hline Frequency of leaning on the elbow & 1 or $3 \dagger$ \\
\hline Magnitude of hand-arm vibration & $1-3 \ddagger$ \\
\hline Frequency of impact shocks during HAV & 1 or $3 \S$ \\
\hline
\end{tabular}

${ }^{*} 1=$ low, $2=$ moderate, $3=$ high.

$+1=$ no, $3=$ yes.

$\ddagger 1=$ none, $2=$ =acceptable, $3=$ high.

$\S 1=$ rare, $3=$ often.

JEM, job exposure matrix.

RNE cases in each occupational group. Ratings were compared and discussed by the experts to resolve any disagreements. A single expert performed the vibration ratings for each occupational group. Ratings were made for all occupational groups except for 'other work'. ${ }^{10}$

Exposure estimates were assigned to individuals based on the JEM ratings for the occupational group corresponding to the job title reported at the last health examination (if more than one examination was available). JEM ratings for each occupational group and biomechanical factor are presented in the online supplementary table 1 . Spearman correlation coefficients were determined between all pairs of biomechanical exposure factors. Highly correlated biomechanical factors (Spearman correlation coefficients $>0.65$; see the online supplementary table 2 ) were summed into biomechanical exposure scores as follows:

$$
\begin{aligned}
\text { Grip Score }= & \text { Intensity of hand grip force }+ \text { Intensity of } \\
& \text { upper extremity load }+ \text { Frequency of hand } \\
& \text { tool use }+ \text { Frequency of using a hand tool } \\
& \text { in a fixed posture (range } 4-12)
\end{aligned}
$$

$$
\begin{aligned}
\text { Vibration Score }= & \begin{array}{l}
\text { Magnitude of HAV + Frequency of impact } \\
\text { shocks during HAV (range 2-6) }
\end{array}
\end{aligned}
$$

Repetitive Flex\&Ext Score $=$ Frequency of repetitive elbow flexion\&extension work + Frequency of repetitive wrist flexion extension work (range 2-6)

Static work/leaning on elbows $=$ Frequency of upper extremity static work + Frequency of leaning on the elbow

Vibration-exposed workers were also likely handgrip exposed as the use of a vibrating hand tool requires grip force; however, these scores were kept separate because they may involve different pathological mechanisms.

Self-reported smoking status was extracted from the same health examination that provided job title, and workers were classified into never, ever and unknown categories. Height and weight were taken from the first health examination; workers were classified into normal (body mass index [BMI] $<25 \mathrm{~kg} / \mathrm{m}^{2}$ ) and overweight (BMI $\geq 25 \mathrm{~kg} / \mathrm{m}^{2}$ ) groups. 


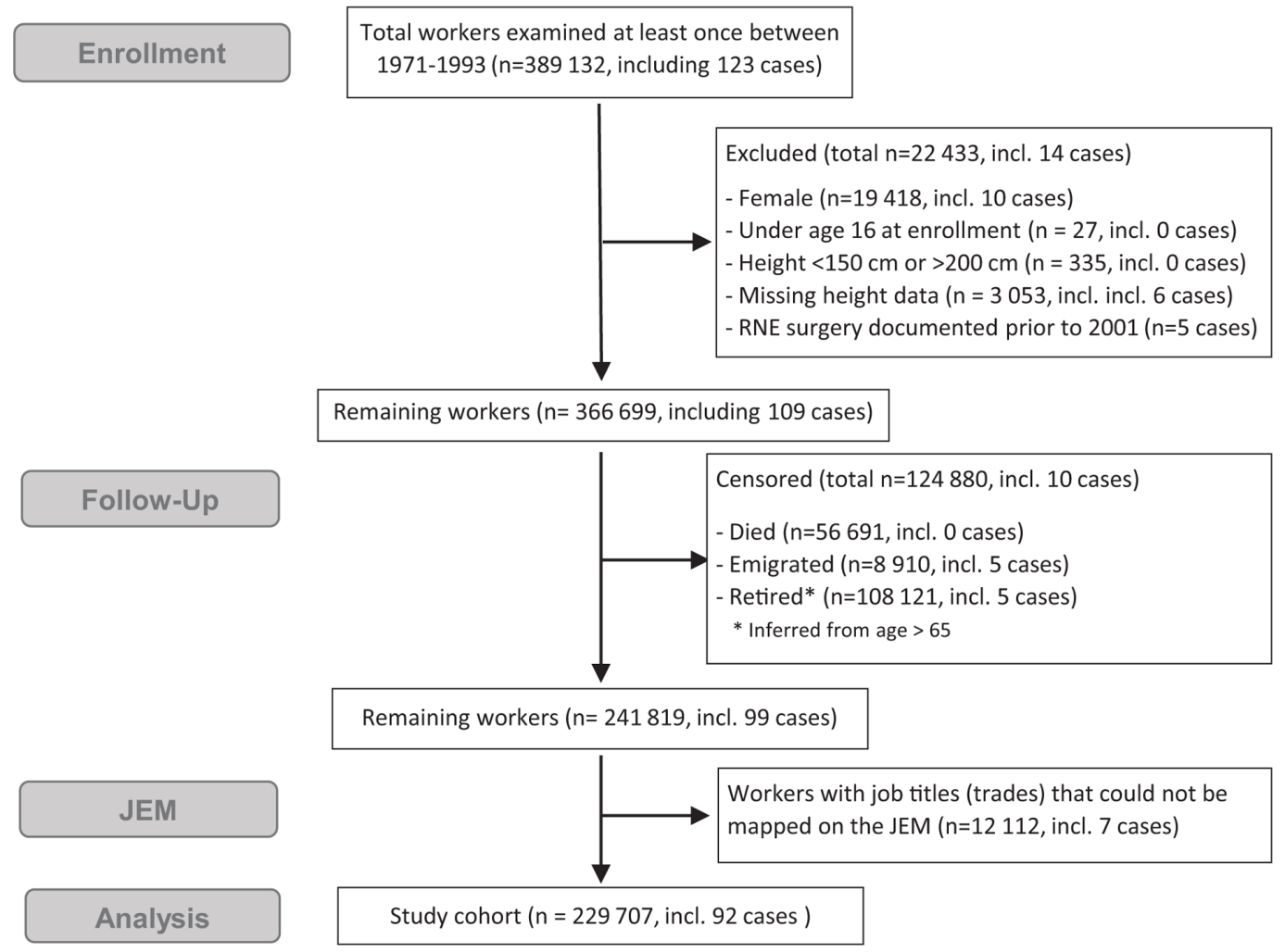

Figure 1 Flow diagram outlining exclusions from construction worker cohort. A single worker may have met more than one criterion within each of the exclusion boxes. JEM, job exposure matrix; RNE, radial nerve entrapment.

In this study, we limited our analysis to men since women comprised only $5 \%$ of the population and most belonged to the 'other' occupational group for whom no physical exposure estimates could be made. Workers were also excluded who: were younger than 16 at their first health examination; were unusually short $(<150 \mathrm{~cm})$ or tall $(>200 \mathrm{~cm})$; or had died, emigrated, retired or had a record of RNE release surgery prior to the start of the observation period in 2001. In addition, workers for whom no job title was recorded in any of the medical examinations or who were classified in the non-specific other work group were removed as they could not be mapped onto the JEM. The remaining 229707 workers comprised the study cohort (figure 1).

\section{Statistical analysis}

Incidence rates of RNE release surgery were calculated for all male construction workers followed through 2013 (figure 1; 99 cases); person-years were calculated from 2001 until date of RNE release surgery, or until the end of the observation period (31 December 2013), censoring for death, emigration or retirement (inferred from age $=65$ ). For comparison purposes, annual RNE surgical release incidence rates were calculated for the Swedish male population aged $25-74$ years (to allow for the 10 -year observation period) based on the National Board of Health and Welfare (Socialstyrelsen) register for the years 2005-2016 (data unavailable prior to 2005). ${ }^{11}$

For the study cohort (92 cases), negative binomial models with a log link were used to estimate relative risks (RR) (incidence rate ratios) and 95\% confidence intervals for the biomechanical factors and biomechanical exposure sum scores as categorical variables, adjusted for age, BMI, smoking status and surgical time (first or second half of the observation period). In all models, the lowest factor or exposure score rating was used as the reference category. The same regression function was used with splining to model incidence for each exposure score as a continuous variable over the range of available values (ranges given for each in equations (1)-(4)) and assess exposure-response relationships.

In order to separate the influence of grip force from hand-arm vibration, a sensitivity analysis was conducted on the association between grip force and RNE decompression surgery only in workers who were not deemed exposed to HAV.

\section{RESULTS}

A total of 99 RNE surgical cases were identified during the observation period, of which 92 cases could be mapped to the 21 occupational groups included in the JEM. The 99 cases represented an incidence rate (IR) of 3.53 cases per 100000 person-years. The rate was higher during the first half of the observation period (2001-2006) compared with the second half (2007-2013; 5.03 vs 3.03 per 100000 person-years).

The concurrent (2007-2013) incidence rate of RNE decompression surgery for men of a similar age range (25-74 years) in the general Swedish population was 2.29 per 100000 personyears (95\% CI 2.09 to 2.51). Thus, the incidence rate in the construction cohort during the second half of the observation period was approximately 30\% higher than the national background rate.

The study cohort $(n=92)$ accrued 2899329 person-years of observation. RNE surgery risk increased with age up until 54 years and then decreased (table 2). No strong association was found between BMI or smoking status and risk of RNE surgery.

Biomechanical factors related to upper extremity loading, elbow posture, vibration, and repetitive and static work were associated with RNE surgical treatment (see the online 
Table 2 Individual factors and RR for RNE decompression surgery $(\mathrm{n}=92)$ from crude models

\begin{tabular}{|c|c|c|c|c|c|c|}
\hline Factor & $N$ & Person-years & Cases & IR & $\mathbf{R R}$ & $95 \% \mathrm{Cl}$ \\
\hline \multicolumn{7}{|l|}{ Individual factors } \\
\hline \multicolumn{7}{|l|}{ BMI } \\
\hline Normal & 167884 & 2128662 & 66 & 3.10 & 1.00 & - \\
\hline Overweight & 61823 & 770667 & 26 & 3.37 & 1.09 & 0.69 to 1.71 \\
\hline \multicolumn{7}{|l|}{ Smoking } \\
\hline Never & 103511 & 1325534 & 37 & 2.79 & 1.00 & - \\
\hline Ever & 115040 & 1435386 & 53 & 3.69 & 1.32 & 0.87 to 2.01 \\
\hline Unknown & 11156 & 138409 & 2 & 1.44 & 0.52 & 0.13 to 2.15 \\
\hline \multicolumn{7}{|l|}{$\begin{array}{l}\text { Age in } \\
2001 \text {, years }\end{array}$} \\
\hline $25-34$ & 32486 & 419152 & 9 & 2.15 & 1.00 & - \\
\hline $35-44$ & 64126 & 824222 & 29 & 3.52 & 1.64 & 0.78 to 3.46 \\
\hline $45-54$ & 73305 & 928305 & 41 & 4.42 & 2.06 & 1.00 to 4.23 \\
\hline $55-64$ & 59790 & 727650 & 13 & 1.79 & 0.83 & 0.36 to 1.95 \\
\hline
\end{tabular}

$I R$, incidence rate per 100000 person-years; $N$, number workers; RNE, radial nerve entrapment; RR, relative risk.

supplementary table 3). An exposure-response relationship was suggested for grip force, upper extremity load, frequency of repeptitive elbow flexion and extension, frequency of repetitive wrist flexion and extension, frequency of hand-held tool use, and exposure to hand-arm vibration.

Biomechanical exposure scores comprising correlated factors showed workers with elevated scores for grip $(R R=1.79)$ and vibration $(\mathrm{RR}=1.47)$ showed the highest risk of $\mathrm{RNE}$ surgery (table 3 ). Workers with exposure to repetitive flexion and extension and static work and elbow leaning had a moderately increased RR for RNE surgery (1.3-fold to 1.4-fold). In the models with continuous exposure scores, both grip $(\mathrm{p}=0.02)$ and repetitive flexion and extension $(\mathrm{p}=0.02)$ were positively associated with RNE surgery incidence (figure 2). No exposureresponse relationship was observed for the vibration or static work and elbow leaning scores.

The sensitivity analysis showed that the RR for exposure to elevated grip force did not differ between workers exposed and unexposed to vibration.

Of the 229707 workers in the study cohort, 150467 participated in at least two health examinations. Of those, $23 \%$ who first reported job titles that mapped to our 21 occupational groups reported a job title on their last health examination that mapped to a different occupational group or the 'other' group. The average time between health examinations was 9.5 years (range: 7.6-11.2 years across occupational groups).

\section{DISCUSSION}

This large cohort of construction workers had an incidence of 3.53 cases per 100000 person-years $(95 \%$ CI 2.88 to 4.28$)$ of surgical release for RNE during the period 2001-2013. Surgical treatment for RNE was associated with work exposure to the biomechanical factors upper extremity load, forceful handgrip work, vibration, and repetitive elbow and wrist flexion and extension, with RRs ranging from 1.5 to 1.9. The biomechanical exposure scoresmost strongly associated with increased risk for RNE surgery were grip and vibration. Although RNE decompression surgery was a rare event in this cohort, given the prevalence of exposure to high grip force (46\%) and the estimated RR of 1.96 , approximately $30 \%$ of all cases in the study cohort can be attributed to high grip force exposure at work.

In this study, we used a cohort-specific JEM based on detailed descriptions of biomechanical exposures and activities comprising each job title. This is often considered the best available method for retrospective exposure assessment in cohort studies. ${ }^{12}$ In the original evaluations, job descriptions were recorded by ergonomists working in the construction industry who observed workers performing each job during regular site visits between 1970 and 1975 . The reports also contained crude estimates of awkward postures and forceful loads, but in a way that was insufficient to facilitate the comparison of specific biomechanical factors that have since been identified as aetiologically relevant for nerve entrapments such as carpal tunnel syndrome and ulnar and RNE symptoms. Hence, our cohort-specific JEM was created by two raters who re-evaluated the historical sources with current insight into relevant exposure parameters. Biomechanical factors that were not documented in the original ergonomic reports but that have since been deemed aetiologically relevant, for example, supination, ${ }^{5}$ were not evaluated in this study.

Many of the biomechanical factors in the JEM were highly correlated, and thus it was difficult to isolate the influence of each specific factor on surgically treated RNE. Exposure sum scores of highly correlated factors were therefore used in the final stage of the model. Some factors, such as hand-arm vibration and grip force, are intrinsically linked and thus their unique

Table 3 Biomechanical exposure scores and the RR for RNE decompression surgery $(n=92)$ in exposed versus unexposed workers

\begin{tabular}{|c|c|c|c|c|c|c|}
\hline Factor & $\mathrm{N}$ & Person-years & Cases & IR & $\mathbf{R R}$ & $95 \% \mathrm{Cl}$ \\
\hline \multicolumn{7}{|l|}{ Grip Score } \\
\hline Unexposed (4) & 48746 & 612804 & 12 & 1.96 & 1 & - \\
\hline Exposed (5-12) & 180961 & 2286525 & 80 & 3.50 & 1.79 & 0.97 to 3.28 \\
\hline \multicolumn{7}{|l|}{ Vibration Score } \\
\hline Unexposed (2) & 104469 & 1314283 & 33 & 2.51 & 1 & \\
\hline Exposed (3-6) & 125238 & 1585046 & 59 & 3.72 & 1.47 & 1.08 to 2.00 \\
\hline \multicolumn{7}{|c|}{ Repetitive Flexion and Extension Score } \\
\hline Unexposed (2) & 81005 & 1019711 & 27 & 2.65 & 1 & - \\
\hline Exposed (3-6) & 148702 & 1879618 & 65 & 3.46 & 1.31 & 0.83 to 2.05 \\
\hline \multicolumn{7}{|c|}{ Static Work and Elbow Leaning Score } \\
\hline Unexposed (2) & 68712 & 866097 & 22 & 2.54 & 1 & - \\
\hline Exposed (3-6) & 160995 & 2033232 & 70 & 3.44 & 1.36 & 0.84 to 2.19 \\
\hline
\end{tabular}

Models adjusted for individual factors: BMI, smoking, age, and time of surgery.

Exposure sum score ranges given in parentheses. Exposure score definitions are given in equations (1)-(4).

$\mathrm{BMI}$, body mass index; IR, incidence rate per 100000 person-years; $\mathrm{N}$, number workers; RNE, radial nerve entrapment; RR, relative risk. 

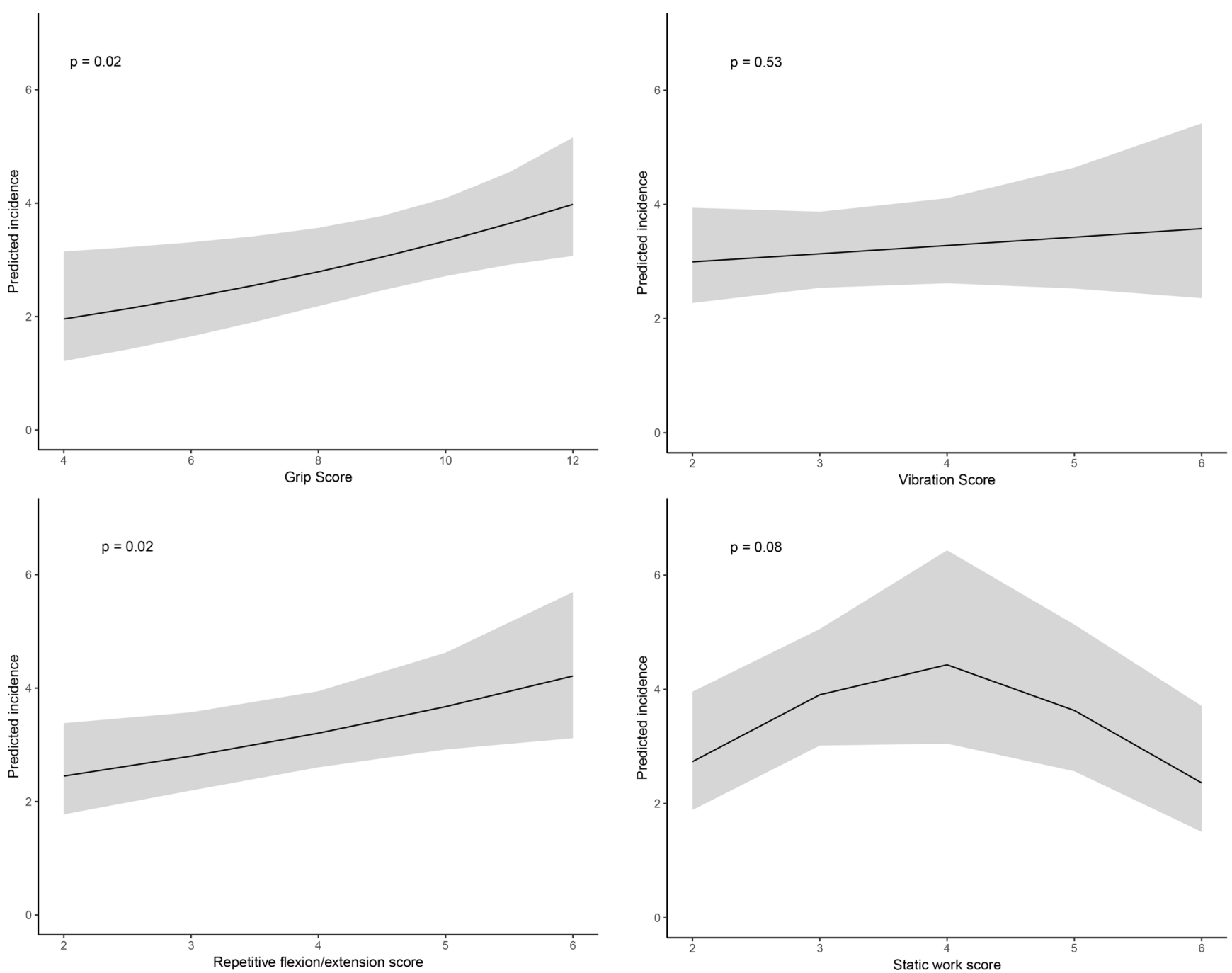

Figure 2 Predicted incidence for onset of RNE decompression surgery by exposure score level modelled as a continuous variable for grip score (unexposed score=4), vibration score (unexposed score=2), repetitive flexion and extension (unexposed score=2) and static work (unexposed score=2). 95\% $\mathrm{Cl}$ shown in grey. RNE, radial nerve entrapment.

contributions cannot be elucidated. The results presented must be viewed as proxies for complex exposure patterns and should not be interpreted as specific exposure-response associations. Further, the study lacks specificity for cumulative exposure level, in absolute terms, or latency period relevant to surgically treated RNE.

The Swedish surgical code used in this study (ACC52) includes decompression surgeries of the radial nerve at all sites in the upper extremity, and not only decompression surgeries distal to the elbow. While decompression surgery is most commonly performed distal to the elbow, some of our cases may include surgeries performed at other locations. Further, decompression surgeries proximal to the elbow can be performed to alleviate both posterior interosseous nerve syndrome (PINS) and radial tunnel syndrome (RTS). While we believe many of the cases in this study were indeed patients with RTS, the diagnosis data in our database are insufficient to quantify this supposition, and thus, the associations shown in the study must be considered at the broader level of 'risk for RNE decompression surgery' rather than for one specific underlying diagnosis, namely, RTS.

Three previously identified biomechanical risk factors in an RTS case-control study ${ }^{5}$ were corroborated in the present study. The finding of forceful handgrip work as a risk factor for RNE is compatible with a pooled analysis of six prospective CTS incidence studies ${ }^{13}$ that identified forceful hand exertions and forceful repetitions as relevant biomechanical exposures. Forceful handgrip work was recently identified as a risk factor for ulnar nerve entrapment in the same Swedish construction cohort. ${ }^{10}$ Pressure on the nerve ${ }^{14}$ has been proposed as the biomechanical mechanism linking high grip force to neuropathy symptoms, such as those experienced in RTS and other compression syndromes.

This study is the largest prospective cohort study to date to assess occupational risk factors for RNE. We were able to identify incident RNE cases and provided ample statistical power while maintaining a strict case definition of physician diagnosis and surgical treatment. The case definition was important given the relative ease of diagnostic confusion between RTS and epicondylitis. ${ }^{15}$ All construction workers within the cohort had good access to occupational healthcare and general medical healthcare, which is nearly free of charge in Sweden. Admission standards may have varied slightly across the country, and construction workers in this nationwide study lived, worked and were treated locally; however, we do not expect that the likelihood of admission would be related to any of the individual or biomechanical exposure variables. Smoking and obesity are 
associated with socioeconomic status; we controlled for these factors in our analyses even in the absence of significant associations with RNE. Last, the consistency of the findings between the categorical and continuous treatment of the exposure scores demonstrates that the results do not rest heavily on the modelling approach chosen.

In this study, occupational biomechanical exposures were determined at the group level, which does not account for individual work strategies or specific job assignments. Further, we did not adjust for potential changes in the occupational group between the health examination at which the job title was recorded until RNE surgery, censoring due to death or immigration, or the end of the observation period. In Sweden, a large proportion of those employed in the construction industry are skilled workers who have rather high-income levels compared with other Swedish blue-collar jobs. Accordingly, workers tend to stay in their trade in the construction industry. While it is likely that some workers changed jobs, and also that the jobs themselves would have changed over time due to different work methods and advancing technologies; this was not reflected in the JEM scores. Finally, individuals who were sensitive to RNE may have changed jobs earlier in their careers, resulting in an over-representation of non-sensitive persons among the highly exposed jobs given the wide range of ages at first examination (16-65 years). This healthy survivor effect would also tend towards an attenuated estimate of the risk.

The incidence rate for RNE decompression surgery for the construction worker cohort was approximately $30 \%-40 \%$ higher than the incidence rate for all Swedish men of the same ages. This further suggests that even the 'low exposed' workers were at elevated risk, compared with their counterparts in jobs outside of the construction sector. If the available dataset had included a truly unexposed reference group, with no history of strenuous manual work, the estimated risk ratios might have been even higher.

Case ascertainment based on surgical treatment can be viewed as both a strength and weakness of the study. The strict criteria were used to avoid misclassification between RTS and epicondylitis, but they may also have resulted in some underestimation of the proportion of workers affected by RNE. Finally, the high grip force requirements present in many construction occupations may have led to a higher incidence of surgical treatment, while workers exposed to lower physical demands may have been more likely to pursue non-surgical treatments.

The association shown in this study between elevated biomechanical exposure and elevated risk for RTS may reflect both risk factors for the onset of RNE and prognostic factors for workers who had previously developed symptoms. Decreasing forceful handgrip and vibration exposure is therefore salient for primary prevention as well as secondary and tertiary prevention of RNE. This recommendation of reduced mechanical loading is in line with the broader ergonomic literature on preventing work-related upper extremity disorders. ${ }^{16} 17$

\section{CONCLUSION}

This large-scale prospective study indicates that biomechanical exposure to forceful handgrip work, vibration, and repetitive elbow and wrist flexion and extension are risk factors for radial nerve decompression surgery.

Acknowledgements The authors thank Hans Petterson for his work in developing the vibration JEM and help in translating job titles, and Per Liv for his assistance with statistical analyses. The Swedish Research Council for Health, Working Life and Welfare supported this study (grant 2016-01016).

Contributors JJ: worked on study design, data analysis, manuscript preparation, revision and submission. DO: worked on data extraction and analysis. AB: worked on study design, and manuscript preparation and revision. LP and JW: worked on study design and manuscript revision. BJ: worked on study design, data analysis and manuscript revision.

Funding This study was funded by Forskningsrådet om Hälsa, Arbetsliv och Välfärd (FORTE 2016-01016).

Competing interests None declared.

Ethics approval The study was approved by the Regional Ethical Review Board in Umeå (2017/16-31).

Provenance and peer review Not commissioned; externally peer reviewed.

Open access This is an open access article distributed in accordance with the Creative Commons Attribution Non Commercial (CC BY-NC 4.0) license, which permits others to distribute, remix, adapt, build upon this work non-commercially, and license their derivative works on different terms, provided the original work is properly cited, appropriate credit is given, any changes made indicated, and the use is non-commercial. See: http://creativecommons.org/licenses/by-nc/4.0/.

\section{REFERENCES}

1 Lister GD, Belsole RB, Kleinert HE. The radial tunnel syndrome. J Hand Surg Am 1979;4:52-9.

2 Latinovic R, Gulliford MC, Hughes RA. Incidence of common compressive neuropathies in primary care. J Neurol Neurosurg Psychiatry 2006;77:263-5.

3 Bolster MA, Bakker XR, Tivente S. Radial tunnel syndrome: emphasis on the superficial branch of the radial nerve. J Hand Surg Eur Vol 2009;34:343-7.

4 Younge DH, Moise P. The radial tunnel syndrome. Int Orthop 1994;18:268-70.

5 Roquelaure Y, Raimbeau G, Dano C, et al. Occupational risk factors for radial tunnel syndrome in industrial workers. Scand J Work Environ Health 2000;26:507-13.

6 Atroshi I, Johnsson R, Ornstein E. Radial tunnel release. Unpredictable outcome in 37 consecutive cases with a 1-5 year follow-up. Acta Orthop Scand 1995;66:255-7.

7 Konjengbam M, Elangbam J. Radial nerve in the radial tunnel: Anatomic sites of entrapment neuropathy. Clinical Anatomy 2004;17:21-5.

8 Roles NC, Maudsley RH. Radial tunnel syndrome: resistant tennis elbow as a nerve entrapment. J Bone Joint Surg Br 1972;54:499-508.

9 Bergdahl IA, Torén K, Eriksson K, et al. Increased mortality in COPD among construction workers exposed to inorganic dust. Eur Respir J 2004;23:402-6.

10 Jackson JA, Olsson D, Punnett L, et al. Occupational biomechanical risk factors fo surgically treated ulnar nerve entrapment in a prospective study of male construction workers. Scand I Work Environ Health 2019:45:63-72.

11 Socialstyrelsen database. Surgery code - ACC52; Population - males ages 25-74 2005-2016 (number surgical patients). http://www.socialstyrelsen.se/statistik/ statistikdatabas/operationerispecialiseradoppenvarddagkirurgi

12 t Mannetje A, Fevotte J, Fletcher T, et al. Assessing exposure misclassification by expert assessment in multicenter occupational studies. Epidemiology 2003:14:585-92.

13 Harris-Adamson C, Eisen EA, Neophytou A, et al. Biomechanical and psychosocial exposures are independent risk factors for carpal tunnel syndrome: assessment of confounding using causal diagrams. Occup Environ Med 2016;73:727-34.

14 Werner CO, Ohlin P, Elmqvist D. Pressures recorded in ulnar neuropathy. Acta Orthop Scand 1985:56:404-6.

15 Neal S, Fields KB. Peripheral nerve entrapment and injury in the upper extremity. Am Fam Physician 2010;81:147-55.

16 da Costa BR, Vieira ER. Risk factors for work-related musculoskeletal disorders: A systematic review of recent longitudinal studies. Am J Ind Med 2010;53:285-323.

17 van der Molen HF, Foresti C, Daams JG, et al. Work-related risk factors for specific shoulder disorders: a systematic review and meta-analysis. Occup Environ Med 2017;74:745-55. 